\title{
POSIÇÃO ESTRATIGRÁFICA DOS EVAPORITOS DA BACIA DE SERGIPE - ALAGOAS
}

\author{
NAMIO UESUGUI*
}

\begin{abstract}
Palynological studies carried out in saliferous sequence of the Sergipe-Alagoas Basin in northeastern Brazil, confirm that the evaporites have been deposited in two distinct sedimentary cycles. The first one, Paripueira Evaporites, was deposited during the Early and Middle Alagoas (local stage) and, the second one, the Ibura Evaporites, during the Late Alagoas. These cycles were previously recognized by means of lithological and stratigraphical studies. On the african Atlantic margin, evaporites contemporaneous with the Paripueira Evaporites have not yet been detected. African evaporites dated as Late Aptian, probably correspond in age to the Ibura Evaporites in Sergipe-Alagoas.
\end{abstract}

INTRODUÇÃo A Formação Muribeca, na Bacia de Sergipe-Alagoas, foi subdividida por Schaller (1969) em cinco membros, aqui enumerados pela ordem de deposição: Maceió, Tabuleiro dos Martins, Carmópolis, Ibura e Oiterinhos. As camadas salíferas ocorrem nos membros Maceió e Ibura e foram designadas, respectivamente, Evaporitos Paripueira e Evaporitos Ibura (Ojeda 1.981, 1982).

Entretanto, a falta de datação segura desses estratos salíferos tem-lhes dificultado a correlação e a colocação na coluna estratigráfica. Com o objetivo de datá-los, efetuou-se estudo palinológico de detalhe em sete poços previamente selecionados com base na boa representatividade em certas áreas da bacia (Fig. 1).

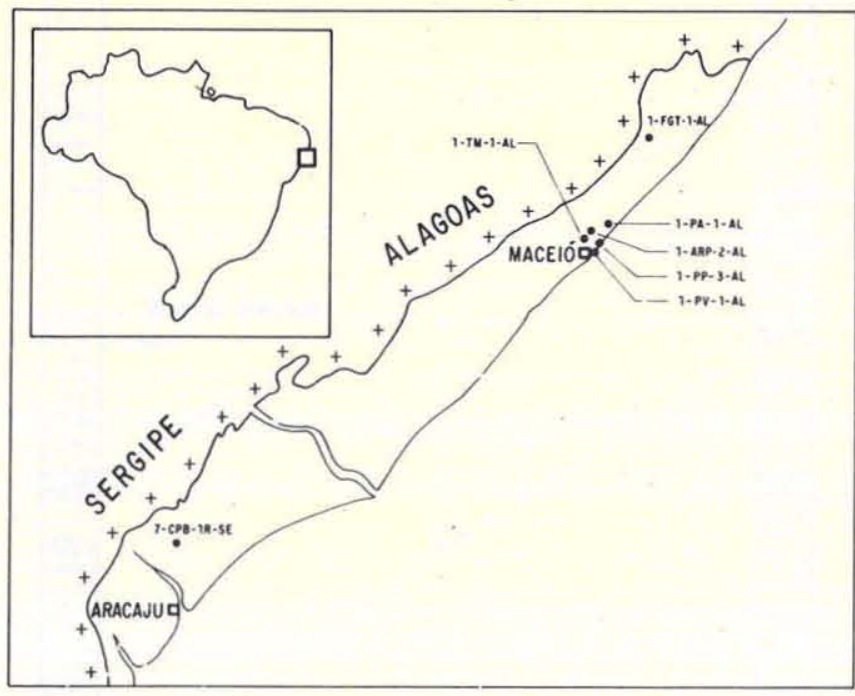

Figura 1 - Mapa de localização dos poços

A figura 2 mostra coluna estratigráfica onde pode ser visualizada a posição da Formação Muribeca.

BIOESTRATIGRAFIA Nos poços analisados 1-ARP-2-AL， 7-CPB-1R-SE，1-FGT-1-AL，1-PA-1-AL, 1-PP-3-AL, 1-PV-1-AL e 1-TM-1-AL -, tomando-se como

\begin{tabular}{|c|c|c|c|c|}
\hline \multicolumn{2}{|c|}{ TEMPO / ANDAR } & LITOLOGIA & FORMACAO & \multirow{2}{*}{$\begin{array}{l}\text { SEQUENNCIA } \\
\text { COBERTURA }\end{array}$} \\
\hline \multirow{2}{*}{ T } & HOLOCENO & Hivivin & $\begin{array}{c}\text { S.P.A } \\
\text { BARREIRAS }\end{array}$ & \\
\hline & \multirow{3}{*}{$\begin{array}{c}\text { MIOCENO } \\
\text { A } \\
\text { ALBIANO }\end{array}$} & & PIAÇABUÇU & \multirow{3}{*}{ MARINHA } \\
\hline SUP. & & & COTINGUIBA & \\
\hline - & & & RIACHUELO & \\
\hline \multirow{3}{*}{ INF. } & AL AGOAS & 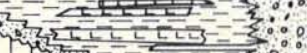 & MURIBECA & $\begin{array}{l}\text { PROTO- } \\
\text { OCEANO }\end{array}$ \\
\hline & JIQUIA' & & $\cos .5$ & \multirow{4}{*}{ RIF TE } \\
\hline & & & 次. CHAVES & \\
\hline \multirow{2}{*}{$k$} & $\begin{array}{l}\text { BURACICA } \\
\text { ARATU }\end{array}$ & & $\begin{array}{ll}\propto & \text { PENEDO } \\
\end{array}$ & \\
\hline & R. SERRA & & B. ITIUBA & \\
\hline \multirow{2}{*}{\multicolumn{2}{|c|}{ JURÁSSICO }} & $\because$ & SERRARIA & \multirow{3}{*}{ PRÉ - RIFTE } \\
\hline & & & $\begin{array}{l}\text { BANANEIRAS } \\
\text { CANDEEIRO }\end{array}$ & \\
\hline \multicolumn{2}{|c|}{ PALEOZÓICO } & 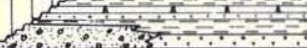 & $\begin{array}{l}\text { ARACARE } \\
\text { BATINGA }\end{array}$ & \\
\hline
\end{tabular}

Figura 2 - Quadro Estratigráfico generalizado da Bacia de Sergipe-Alagoas (adaptado de Schaller et al. 1980)

base o zoneamento bioestratigráfico de Regali et al. (1974), foram identificadas as seguintes zonas: P-230 (Inaperturopollenites crisopolensis), P-260 (Inaperturopollenites turbatus), P-270 (Sergipea variverrucata) e P-280 (Caytonipollenites sp.1).

As zonas P-240 e P-250 não foram utilizadas neste trabalho devido à baixa ocorrência das espécies-guia.

$\mathrm{Na}$ tabela 1 estão listados os topos das quatro zonas e as respectivas profundidades.

POSIÇÃO ESTRATIGRÁFICA DO SAL As quatro zonas palinológicas identificadas foram rastreadas nos poços estudados e associadas à coluna litológica esquemática de cada um deles. Observando-se as figuras 3 e 4, verifica-se que os Evaporitos Paripueira iniciaram sua deposição dentro da zona P-230, parte superior, estendendo-se até o topo da zona P-260 (poços 1-FGT-1-AL e 1-PA-1-AL); no poço 1-ARP-2-AL ocorrem somente no intervalo equivalente ao topo da zona P-260. Nos poços 1-TM-1-AL, 1-PP-3-AL e 1-PV-1-AL, embora as zonas P-230 e P-260 estejam presen- 
Tabela 1 - Topo das zonas nos sete poços

\begin{tabular}{l|c|c|c|c}
\hline \multicolumn{1}{c|}{ Poço } & $\begin{array}{c}\text { Zona P-230 } \\
(\mathrm{m})\end{array}$ & $\begin{array}{c}\text { Zona P-260 } \\
(\mathrm{m})\end{array}$ & $\begin{array}{c}\text { Zona P-270 } \\
(\mathrm{m})\end{array}$ & $\begin{array}{c}\text { Zona P-280 } \\
(\mathrm{m})\end{array}$ \\
\hline 7-CPB-1R-SE & - & - & 420 & 260 \\
1-ARP-2-AL & 1.770 & 1.230 & 390 & - \\
1-FGT-1-AL & 2.380 & 1.650 & 30 & - \\
1-PA-1-AL & 2.240 & 1.490 & 160 & - \\
1-PP-3-AL & 1.690 & 1.040 & 390 & - \\
1-PV-1-AL & 1.030 & 740 & 140 & - \\
1-TM-1-AL & 990 & 520 & 190 & - \\
\hline
\end{tabular}

tes, não houve a deposição de sal. Pelo visto, os Evaporitos Paripueira representaram vários episódios, cujo ciclo deposicional completo se iniciou na zona P.230 e terminou na P-260. Sua presença ou ausência, entretanto, depende da posição do poço em relaçẫo às condições paleogeográficas da época.
Nos poços 1-TM-1-AL e 7-CPB-1R-SE ocorrem os Evaporitos Ibura, que estão restritos à parte superior da zona P-270. Estes dois poços representam o elo de ligação dos Evaporttos Ibura nos dois lados da bacia, evidenciando, também, a nítida separação dos dois ciclos evaporíticos, separados por um espesso pacote de clásticos.

CORRELAÇÓES COM OS SAIS AFRICANOS Ao confrontar as zonas palinológicas da Bacia de Sergipe-Alagoas (Regali et al. 1974) com as do Gabão (Doyle et al. 1982), notamos a perfeita correspondência da zona P-230 com a C-VII, pois ambas foram definidas pelo desaparecimento da espécie-guia Inaperturopollenites crisopolensis. Os Evaporitos Paripueira iniciaram seu ciclo deposicional nesta época. $\mathrm{Na}$ África, não foram encontrados evaporitos até a presente data; registrou-se apenas um aumento de concentração de lítio e boro nas análises geoquímicas das amostras do centro e sul do Gabão, indicando, no máximo, aumento de salinidade de água neste tempo (Doyle et al. 1982). Esses dados

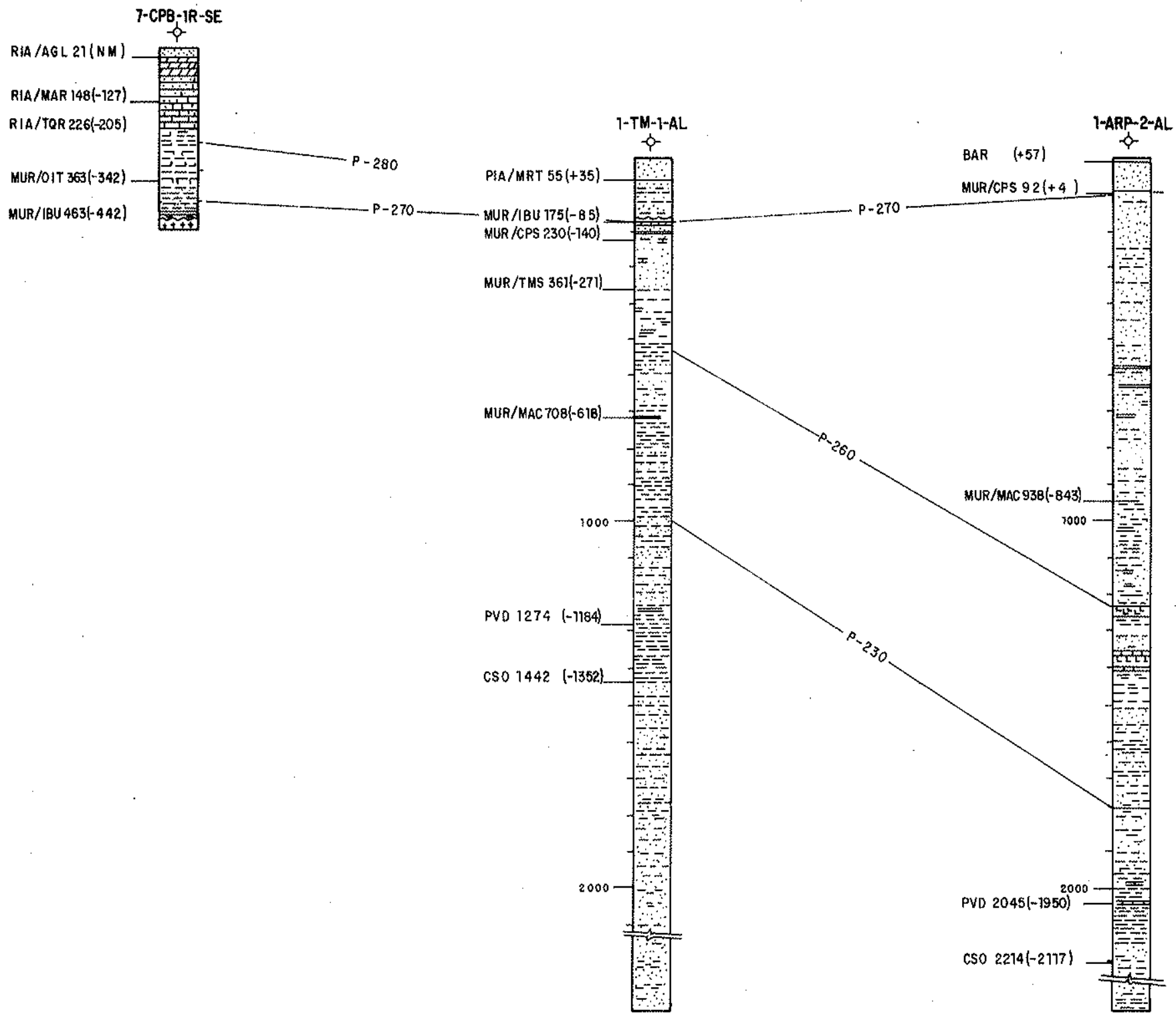

Figura 3 - Correlações das biozonas na seção BB' com um poço de Sergipe (mesma legenda da figura 2) 


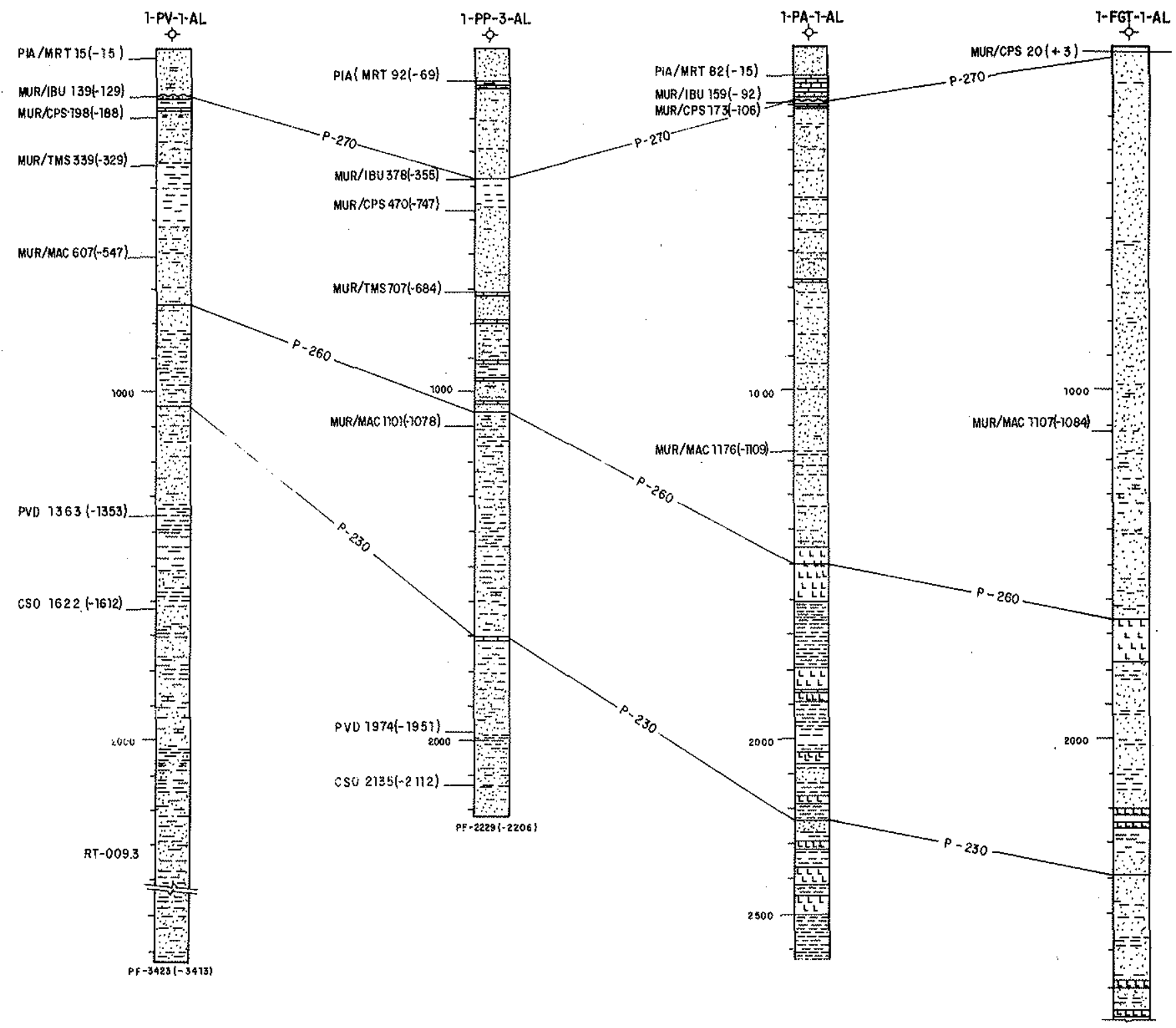

Figura 4 - Correlações das biozonas na seção AA' com um poço de Sergipe (mesma legenda da figura 2)

vêm corroborar a hipótese de Ojeda (1981), de que, na África, evaporitos correspondentes ao Paripueira poderiam ter sido depositados além da atual plataforma continental.

Os primeiros registros de evaporitos africanos ocorrem na zona C-IX (Doyle et al. op. cit.), correspondentes, no tempo, à zona P-270; portanto, equivalentes aos Evaporitos Ibura.

CRONOESTRATIGRAFIA Os Evaporitos Ibura são isentos de fósseis marinhos. Entretanto, a presença de Cheloniceras spp., Hedbergella washitensis praecursor e Nannoconus truitti nos sedimentos sobrejacentes do membro Oiterinhos permite atribuir a idade neoaptiana para esses sedimentos (Ojeda 1981).

$\mathrm{Na}$ África, a Série Madiela é datada de Neoaptiano ao Neoalbiano pela ocorrência de Deshayesites consorbinoides (Neoaptiano) na parte mais basal dos carbonatos, Douvilleiceras monile (Eoalbiano) e Elobiceras szajnochai (Neoal- biano). A recente descoberta de Colomiella erecta, um tintinídeo, na zona palinológica $\mathrm{C}-\mathrm{X}$, no poço Coniquet 1 , na região de Libreville, restrito ao Eoalbiano, vem confirmar a possibilidade de que a parte mais basal desta série seja de idade Neoaptiana (Doyle et al. 1982).

Quanto aos Evaporitos Paripueira sotopostos aos Evaporitos Ibura e separados por uma espessa seção de sedimentos clásticos, foram considerados por Ojeda (1981) de idade eoaptiana. Esta atribuição é bastante precária em função da ausência total de fósseis marinhos nesses sedimentos. $\mathrm{Na}$ África, estratos correspondentes a esses evaporitos não apresentam camadas salíferas. Doyle et al. (op. cit.) atribuem o topo do limite do Eoaptiano no topo da zona C-VII. Este limite foi estabelecido em comparação com o conteúdo fossilifero das rochas marinhas do Gondwana norte, precariamente datadas em relação ao andar-padrão do Eocretáceo. A zona $\mathrm{C}$-VII é equivalente à $\mathrm{P}$-230 de Sergipe-Alagoas. Pelo visto, os limites do Eoaptiano adotado por Doyle et al. (op. 
cit.) na África e no Brasil por Ojeda (op. cit.) não são coincidentes, pois os Evaporitos Paripueira terminaram a deposição no topo da zona P-260. Portanto o limite do Aptiano Superior é mais alto no Brasil.

Em virtude da precariedade da datação dos evaporitos em ambos os lados do Atlântico, em relação aos andares do Eocretáceo, preferimos a datação baseada nos andares locais estabelecidos para as seções não-marinhas das bacias marginais brasileiras. Assim, os Evaporitos Paripueira foram relacionados ao Eo-Mesoalagoas e, os Evaporitos Ibura, ao Neoalagoas.

CONCLUSÕES A palinologia confirma a existência de dois ciclos evaporíticos de idades diferentes na Bacia de Sergipe-Alagoas.

Os Evaporitos Paripueira compõem-se de diversas camadas de sal, ocupando posições estratigráficas diferentes, den- tro de um ciclo deposicional, e depositaram-se no Eo-Mesoalagoas. Os Evaporitos Ibura depositaram-se num ciclo posterior, de curta duração, no Neoalagoas, antes do estabe- lecimento das condições pré-oceânicas do Atlântico Sul mesocretácico.

As correlações dos dados palinológicos da margem brasileira com os da margem africana revelam que exite perfeita correspondência entre os sais em ambos os lados, do ciclo Evaporitos Ibura. Em relação aos Evaporitos Paripueira, na África, embora os sedimentos desta idade estejam presentes, evaporitos deste ciclo ainda não foram encontrados.

Agradecimentos $\mathrm{O}$ autor agradece à Petrobrás, pela publicação deste trabalho; ao geólogo Dimas Dias-Brito, chefe do Sebipe, pelas valiosas sugestões; e a Fani Knoploch, Sintep-Getec, pela revisão do texto.

\section{REFERÊNCIAS BIBLIOGRÁFICAS}

DOYLE, J.A.; JARDINÉ, S.; DOERENKAMP, A. - 1982 - Afropollis, a new genus of early angiosperm pollen, with notes on the Cretaceous palynostratigraphy and paleoenvironments of Northern Gondwana. Bull. Cent. Rech. Prod. Elf-Aquitaine, 6(1):39-117.

OJEDA, H.A.O. - 1981 - Estrutura, estratigrafia e evolução das bacias marginais brasileiras. Rev. Bras. Geoc., 11(4):257-273.

OJEDA, H.A.O. - 1982 - Structural framework, stratigraphy and evolution of brazilian marginal basins. Am. Assoc. Pet. Geol., 66(6):732-749.

REGALI, M.S.P.; UESUGUI, N.; SANTOS, A.S. - 1974 - Palinologia dos sedimentos meso-cenozóicos do Brasil. Bol. Téc. Petrobrás, 17(3):117-191; e 17(4):263-301.
SCHALLER, H. - 1969 - Revisão estratigráfica da Bacia de Sergipe-Alagoas. Bol. Téc. Petrobrás, 12(1):21-86.

SCHALLER, H.; DELLA FÁVERA, J.C.; TIBANA, P. - 1980 -. Roteiro geológico da Bacia de Sergipe-Alagoas. Rio de Janeiro, Petrobrás, (Rel. int.).
MANUSCRITO 388

Recebido em 01 de Setem bro de 1986 Revisão aceita em 02 de abril de 1987

"É pelo convencimento de que a universidade existe, sobretudo, para pensar... que deve iniciar-se o longo caminho de reconstrução da universidade que o País necessita. E é pela mobilização das energias universitárias no esforço de pensar, sem preconceitos, com imaginação e com espírito crítico, que a universidade dará a passo seguinte". 